\title{
Corrigendum
}

\section{Umbilical Cord Structure in a 21-Week Fetus}

\section{Carter E. Wahl}

Eliezer Masliah

Journal of Perinatology (2004) 24, 405. doi:10.1038/sj.jp.7211108

Correction to: Journal of Perinatology (2004) 24, 48-49, doi:10.1038/sj.jp.7211015

Due to an oversight on one of the author's part, the order of the author names was published incorrectly. Dr. Masliah was the first author credited, when the order should be as follows:

Carter E. Wahl, MD

Eliezer Masliah, MD 